\title{
The Alisma and Rhizoma decoction abates nonalcoholic steatohepatitis-associated liver injuries in mice by modulating oxidative stress and autophagy
}

Lijun $\mathrm{Xu}^{1+}$, Menghui Jing ${ }^{1+}$, Lijuan Yang ${ }^{2}$, Lei Jin ${ }^{1}$, Peiqiang Gong ${ }^{3}$, Juan Lu ${ }^{1}$, Hui Lin ${ }^{1}$, Jinping Wang ${ }^{1}$, Qin $\mathrm{Cao}^{1 *}$ and Yuanye Jiang ${ }^{1 *}$

\begin{abstract}
Background: To investigate the effects of the Alisma and Rhizoma decoction on nonalcoholic steatohepatitis (NASH) and to further shed light on the underlying mechanisms of the actions of the Alisma and Rhizoma decoction.

Methods: Plasma alanine aminotransferase (ALT) content was determined and liver inflammation and fibrosis were evaluated. Intrahepatocellular malondialdehyde and superoxide dismutase contents were determined using commercially available kits Furthermore, a-SMA expression in liver tissues was examined by immunohistochemistry and LC3-II was detected by immunoblotting assays.

Results: Mice receiving the Alisma and Rhizoma decoction by gastric lavage had significantly lower plasma ALT content and markedly higher hepatic superoxide dismutase activity than mice receiving the methionine-choline deficient (MCD) diet. Furthermore, the decoction aborted MCD-induced increase in liver malondialdehyde content. Immunohistochemistry showed that the decoction suppressed hepatic a-SMA expression. Our transmission electronic microscopy revealed that the decoction markedly reduced the number of autophagosomes and immunoblotting assays showed that the decoction caused a dose-dependent decrease in LC3-II in hepatic tissues.
\end{abstract}

Conclusion: The Alisma and Rhizoma decoction lessens NASH-associated liver injuries by modulating oxidative stress and autophagy in hepatocytes of mice fed with MCD.

Keywords: Alisma orientale, Rhizoma atractylodis macrocephalae, Non-alcoholic fatty liver disease, Liver inflammation, Immunoblotting assays

\section{Background}

Non-alcoholic fatty liver disease (NAFLD) represents a broad spectrum of liver diseases ranging from simple steatosis to steatohepatitis through to fibrosis and cirrhosis [1] and is characterized by excessive lipid accumulation in the liver without chronic alcohol consumption. The global prevalence of NAFLD is $20-30 \%$, affecting one billion persons worldwide [2]. The prevalence of NAFLD has been steadily increasing in China and has reached

\footnotetext{
* Correspondence: caoqin434@sina.com; jiangyuanye88@163.com ${ }^{\dagger}$ Lijun Xu and Menghui Jing are contributed equally to this work. ${ }^{1}$ Department of Gastroenterology, Putuo Hospital, Shanghai University of Traditional Chinese Medicine, Shanghai 200062, China

Full list of author information is available at the end of the article
}

approximately $15 \%$ in economically developed metropolitan areas [3]. Non-alcoholic steatohepatitis (NASH) is a critical step in the progression of NAFLD and is marked by fatty degeneration of hepatocytes, necrotic inflammation and/or fibrosis and may further progress to hepatic fibrosis and even liver cancer. Liver fibrosis is a common entity underlying various chronic liver diseases and recently it has been shown development of liver fibrosis is a dynamic process and reversible under certain conditions [4].

Currently, the mechanisms underlying NASH liver fibrosis has not been fully elucidated and no effective treatment is available clinically. It has been recently reported that autophagy is implicated in the development

(c) The Author(s). 2019 Open Access This article is distributed under the terms of the Creative Commons Attribution 4.0 International License (http://creativecommons.org/licenses/by/4.0/), which permits unrestricted use, distribution, and reproduction in any medium, provided you give appropriate credit to the original author(s) and the source, provide a link to the Creative Commons license, and indicate if changes were made. The Creative Commons Public Domain Dedication waiver (http://creativecommons.org/publicdomain/zero/1.0/) applies to the data made available in this article, unless otherwise stated. 
and progression of hepatic fibrosis [5-7]. The autophagy flux increased when primary mouse hepatic stellate cells (HSC) became activated, which were suppressed by autophagy inhibitor afilomycin A1 [8]. Furthermore, autophagy-stimulated loss of lipid droplets was shown to modulate HSC activation [9]. These findings together suggest that autophagy may participate in the progression of fibrosis via modulation of HSC activation.

Alisma orientale (Sam.) Juzep. (Alismataceae) is a traditional medicinal herb [10] that has been used in China for treating a variety of conditions including hypertension, hyperlipidemia, Meniere's disease and other conditions and $A$. orientale and its constituents have been shown to possess a broad spectrum of biological activities including anti-atherosclerotic activity, immunomodulation, and hepatoprotection [11-13]. Hong et al. showed that $A$. orientale methanolic extract improved the plasma lipid profile and alleviated hepatic steatosis in rats with high fat diet-induced NAFLD [10]. Jang et al. demonstrated that $A$. orientale attenuated hepatic steatosis of mice with high fat diet-induced obesity by suppressing endoplasmic reticulum (ER) stress. In vitro evidence also showed that $A$. orientale extract inhibited lipogenesis and lipoapoptosis of non-esterified fatty acid-treated HepG2 cells [14]. Alisol B 23-acetate, a natural triterpenoid isolated from Rhizoma Alismatis, the dried rhizome of $A$. orientale, was found to protect against non-alcoholic steatohepatitis in mice by activating the farnesoid X receptor [15]. Rhizoma atractylodis macrocephalae has long been widely used in East Asia as a traditional herbal medicine and constituents from $R$. atractylodis macrocephalae have been shown to possess anti-inflammatory activities in mice [16]. Fermented $R$. atractylodis macrocephalae was found to improve the lipid profile of rats with high fat diet-induced obesity [17].

The ancient Chinese medical scripture "the Inner Canon of the Yellow Emperor" mentions the use of the Alisma and Rhizoma decoction with ten equal parts of $A$. orientale and $R$. atractylodis macrocephalae for treating "alcoholic fever", which manifests as lethargy and fever. Currently, the Alisma and Rhizoma decoction is widely used for the treatment of hyperlipidemia, fatty liver disease and Meniere's syndrome [18-20]. Our previous clinical study found that compared to Western drugs, the Alisma and Rhizoma decoction alleviated liver injury and improved the lipid profile of NAFLD patients, suggesting that the decoction exerts hepatoprotective effects in NAFLD patients. However, the mechanisms whereby the Alisma and Rhizoma decoction exerts its myriad effects have not been elucidated.

We speculated that the Alisma and Rhizoma decoction could alleviate NASH-induced liver injuries via modulation of autophagy. In the current study, we established a mouse NASH model by the methionine-choline deficient (MCD) [21, 22] diet and investigated the effects of the Alisma and Rhizoma decoction on NASH mice and further shed light on the underlying mechanisms.

\section{Methods \\ Animals}

The study protocol was approved by the Experimental Animal Ethical Committee, Putuo Hospital, Shanghai University of Traditional Chinese Medicine. Animal study was carried out in strict accordance with the established institutional guidelines and the NIH guidelines on the use of experimental animals.

Thirty-six 8-week old male C57BL/6 mice weighing $20 \pm 3.5 \mathrm{~g}$ were purchased from the Experimental Animal Center at the Putuo District Central Hospital, Shanghai University of Traditional Chinese Medicine. The animals were housed at a constant temperature $\left(20-22{ }^{\circ} \mathrm{C}\right)$ at 50 $70 \%$ humidity with a $12 \mathrm{~h} \mathrm{light/} \mathrm{dark} \mathrm{photoperiod.} \mathrm{Mice}$ were provided with regular laboratory chow and water ad libitum and were allowed 1 week to accommodate.

\section{Treatments}

A. orientale granules and $R$. atractylodis macrocephalae granules were identified and authenticated by the Traditional Chinese Medicine Pharmacy of Putuo District Central Hospital. The specimen voucher number is A1500730 for A. orientale granules and A1501391 for $R$. atractylodis macrocephalae granules. The stock was prepared at a ratio of $5(A$. orientale):2 ( $R$. atractylodis macrocephalae) for gastric lavage. The mice were randomized to receive the regular diet and $2 \mathrm{~mL}$ distilled water (group I, $n=12$ ), the MCD (Trophic Animal Feed High-Tech Co., Nantong, China) and $2 \mathrm{~mL}$ distilled water (group II, $\mathrm{n}=12$ ), or MCD and $2 \mathrm{~mL}$ the Alisma and Rhizoma decoction $(4.31 \mathrm{~g} / \mathrm{kg}$ ) (group III, $\mathrm{n}=12)$ by gastric lavage for 12 weeks.

\section{Biochemical determinations}

Mice were anesthetized with $2 \%$ sodium pentobarbital $(75 \mathrm{mg} / \mathrm{kg})$ intraperitoneally. Venous blood was obtained via the orbital vein and centrifuged at $3000 \mathrm{rpm}$ for 10 min. The animals were euthanized by cervical dislocation. Liver tissues were obtained via abdominal dissection. The above information is incorporated in the revised manuscript. The supernatant was saved and analyzed using a Vitros350 Automatic Biochemical Analyzer (Johnson \& Johnson) for plasma alanine aminotransferase (ALT) content.

\section{Hematoxylin and eosin (H\&E) staining}

At the end of 12 week treatment by gastric lavage, the animals were sacrificed by cervical dislocation and liver tissues were obtained via abdominal dissection and fixed 
in $4 \%$ paraformaldehyde and paraffin embedded. Serial sections were sliced at a thickness of $5 \mu \mathrm{m}$ (Lecia, Wetzlar, Germany). Tissue sections were stained with $H \& E$ using the standard protocol. Briefly, the sections were stained in hematoxylin for $10 \mathrm{~min}$, followed by staining in $1 \%$ eosin solution for $3 \mathrm{~min}$, and subsequently washed with distilled water. The slides were then observed under a light microscope (BX51, Olympus Co.) for fatty changes of the liver, hepatic inflammation and fibrosis. NAFLD activity score (NAS) (ranging 0-8) was assessed as follows: 1) a score of 0 represented $<5 \%$ fatty degeneration of liver cells, 1 represented 5-33\%, 2 $34-66 \%$, and 3 represented $>66 \%$ fatty liver degeneration; 2) a score of 0 represented lack of lobular inflammation (number of necrotic foci under $20 \times$ microscope), 1 indicated the number of necrotic foci $<2$; 2 indicated 2-4 necrotic foci, and 3 indicated $>4$ necrotic foci; (3) a score of 0 and 1 represented no or scant balloon like changes of hepatocytes, and 2 indicted more balloon like changes. Each subscore was added and the total mean score was reported. $\mathrm{NASH}$ was excluded if NAS was $<3$ and NASH was diagnosed if NAS was $>4$. Liver inflammation and fibrosis were determined as previously described [23]: G0 indicates no inflammation; G1 mild inflammation; G2 moderate inflammation; G3 moderate-to-severe inflammation; G4 severe inflammation; S0 represents no fibrosis; S1 portal fibrosis without septa; S2 star-shaped portal fibrosis, with minimal septa; S3 portal fibrosis with numerous septa, but no pseudo lobe formation; S4 portal fibrosis with numerous septa and pseudo lobe formation.

\section{Immunohistochemistry}

Immunohistochemistry was performed using the universal two-step immunohistochemical method. The expression of $\alpha$-SMA in liver tissues was examined was detected using rabbit monoclonal antibody against $\alpha$-SMA (catalog \# sc-53,142; dilutions 1:200; Santa Cruz Biotechnology, Santa Cruz, CA). PBS was added instead of the primary antibody in the negative control.

\section{Transmission electron microscopy}

Liver tissue blocks were postfixed in $2.5 \%$ glutaraldehyde for $2 \mathrm{~h}$ at room temperature, and the tissue was embedded in Epon 812. Ultrathin sections (50-80 nm) were cut with an ultramicrotome (Leica RM 2165, Leica Ultracut UCT, Germany) and stained with uranyl acetate and lead citrate. Observations and photomicrographs were made with a transmission electron microscope (TEM; JEOL JEM-2100, Japan) operated at $60 \mathrm{kV}$.

\section{Determination of malondialdehyde and superoxide} dismutase contents

Homogenate of hepatic tissues was prepared as previously described [24, 25]. The tissue homogenate was centrifuged, and the supernatant was collected and stored at $-84^{\circ} \mathrm{C}$. Malondialdehyde and superoxide dismutase contents were determined using commercially available kits (Nanjing Jiancheng Bioengineering Institute, Nanjing, China) as instructed by the manufacturer. Measurements were done at least three times independently and averaged.

\section{Western blotting assays}

Liver tissues were lyzed using RIPA lysis buffer containing a cocktail of protease inhibitors and phosphorylase inhibitors (Roche). Protein content in the lysates was determined by the BCA method. Samples were resolved by $8 \%$ SDS PAGE. Nonspecific binding was blocked with $5 \%$ bovine serum albumin. Anti-LC3-IIantibody (catalog \# sc-292,354; dilutions 1:3000; Santa Cruz Biotechnology) and HRP-conjugated $\beta$-actin (catalog \# HRP-60008; dilutions 1:1000; Proteintech Group, Wuhan, China). Membranes were visualized by using an ECL Plus Western Blotting Detection System (GE Healthcare, USA). Protein densitometry was performed using Image-ProPlus 6.0 image analyzing software (Media Cybe-netics Co.). LC3-II expression was normalized against $\beta$-actin.

\section{Statistical analysis}

Data were expressed as mean \pm standard deviations and analyzed using the SPSS23.0 statistical analysis software (SPSS Inc., Chicago, IL, USA). Comparison of multiple groups was done by ANOVA, and comparison between two groups used Student's $t$-test. $P<0.05$ was considered statistically significant.

\section{Results \\ The Alisma and Rhizoma decoction lessens NASH-like changes in mice}

We first examined the effect of the Alisma and Rhizoma decoction on MCD-induced NASH-like changes in mice. Histological examination showed that group I had normal liver architecture with intact hepatic lobules and with no fatty changes in hepatocytes (S0) (Fig. 1a). Group II showed indistinct lobule border, narrowed sinusoidal space and periportal fibrosis with septa (S2 and S3) (Fig. 1b). Massive punctate necrosis and balloon-like changes were observed, indicating that MCD induced NASH-like changes in these mice. By contrast, group III showed virtually no portal fibrosis and no septa (S1) and less severe balloon-like changes (Fig. 1c), suggesting that the Alisma and Rhizoma 


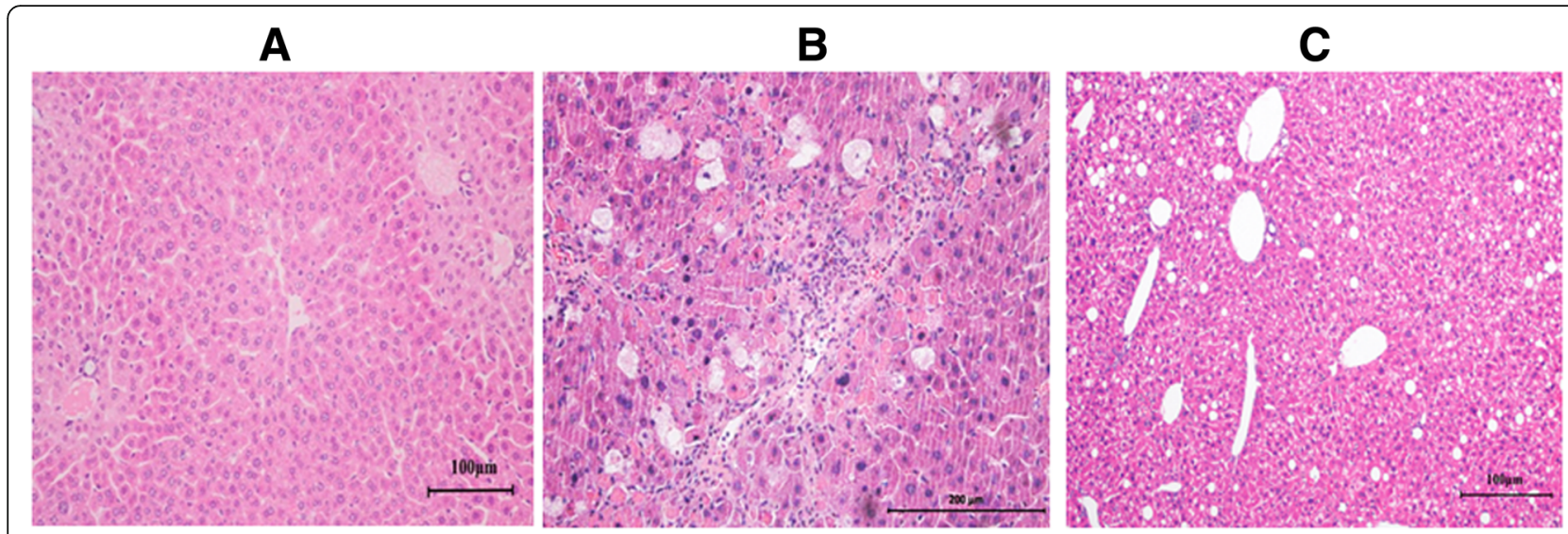

Fig. 1 The Alisma and Rhizoma decoction lessens NASH-like changes in mice. Mice were randomized to receive the regular diet and $2 \mathrm{~mL}$ distilled water (a), the methionine and choline diet (MCD) and $2 \mathrm{~mL}$ distilled water (b), or the MCD and $2 \mathrm{~mL}$ Alisma and Rhizoma decoction $(4.31 \mathrm{~g} / \mathrm{kg})(\mathbf{c})$ by gastric lavage for 12 weeks. H\&E stained mouse liver tissue sections were examined for fatty changes of the liver, hepatic inflammation and fibrosis under a light microscope as described in Materials and methods. Magnification, $100 \times$

decoction attenuated MCD-induced NASH-like changes in mice.

\section{The Alisma and Rhizoma decoction attenuates MCD- induced liver injuries}

We were interested in whether improvement by the Alisma and Rhizoma decoction in liver morphology in mice with MCD-induced NASH-like changes was associated with amelioration in hepatic function. The plasma ALT content in group I was $33.5 \pm 3.9 \mathrm{IU} / \mathrm{mL}$. The plasma ALT content $(51.8 \pm 7.8 \mathrm{IU} / \mathrm{mL})$ in group II was significantly higher than that of group I. This rise in plasma ALT content, however, was significantly attenuated in mice fed with the Alisma and Rhizoma decoction (38.2 $\pm 5.2 \mathrm{IU} / \mathrm{mL})(P<0.01)$ (Fig. 2a).

In addition, the NAS score in group II $(7.00 \pm 1.73$ was significantly higher than that of group I (1.33 \pm 1.53) $(P<0.05)$. The Alisma and Rhizoma decoction markedly attenuated MCD induced increase in NAS score $(P<0.05)$ (Fig. 2b). The above findings suggested that the Alisma and Rhizoma decoction attenuates MCD-induced liver injuries.

\section{The Alisma and Rhizoma decoction reduces oxidative stress in MCD-induced NASH}

It has been reported that the aqueous extract of $A$. orientale protected against long-chain saturated fatty acid-induced cellular injury by attenuating oxidative stress [26]. We also found that the SOD activity of the liver homogenate was $22.67 \pm 11.45 \mathrm{U} / \mathrm{mg}$ protein in group II, which was significantly lower than that of group I $(77.76 \pm 11.33 \mathrm{U} / \mathrm{mg}$ protein $)(P<0.01)$. This decrease, however, was markedly abated by treatment with the Alisma and Rhizoma decoction $(63.14 \pm 14.08$ $\mathrm{U} / \mathrm{mg}$ protein) $(P<0.05)$ (Fig. 3a). Furthermore, the MDA content of the liver homogenate was $6.93 \pm 1.88$ $\mathrm{mmol} / \mathrm{mg}$ protein in group I. MCD caused a significant increase in liver MDA content $(15.65 \pm 2.92 \mathrm{mmol} / \mathrm{mg})$
A

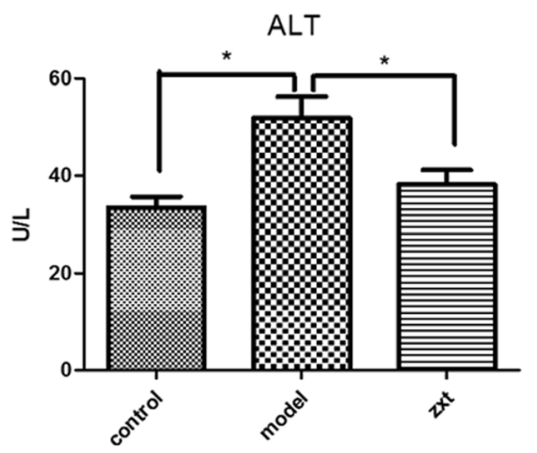

B

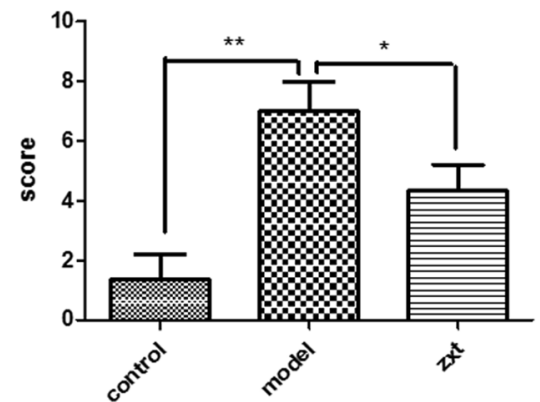

Fig. 2 The Alisma and Rhizoma decoction attenuates MCD-induced liver injuries. Mice were randomized to receive the regular diet and 2 mL distilled water (group I), the methionine and choline diet (MCD) and $2 \mathrm{~mL}$ distilled water (group II), or the MCD and $2 \mathrm{~mL}$ Alisma and Rhizoma decoction $\left(4.31 \mathrm{~g} / \mathrm{kg}\right.$ ) (group III) by gastric lavage. a Serum ALT content in each group. b NAS scores in each group. * $P<0.05,{ }^{* *} P<0.01$ 
A

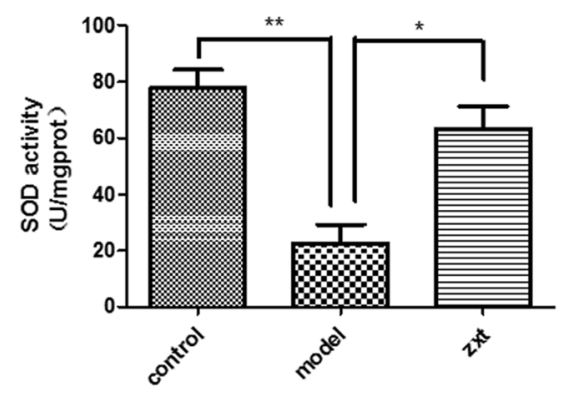

B

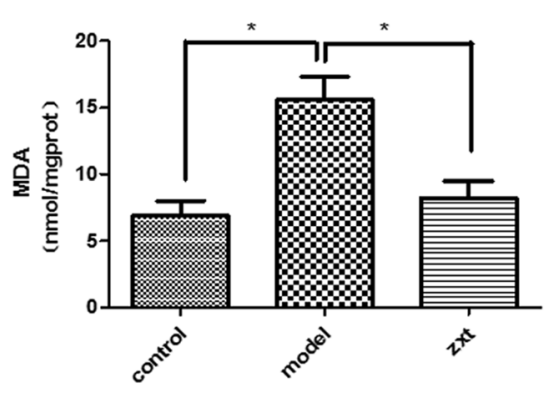

Fig. 3 The Alisma and Rhizoma decoction reduces oxidative stress in MCD-induced NASH. Mice were treated as detailed in Fig. 2. The SOD activity (a) and MDA content (b) of the liver homogenate were measured as described in Methods. ${ }^{*} P<0.05$, ${ }^{*} P<0.01$

$(P<0.05$ versus group I). This increase, however, was aborted by the Alisma and Rhizoma decoction $(8.25 \pm$ $2.16 \mathrm{mmol} / \mathrm{mg})(P<0.05$ versus group II) (Fig. 3b).

\section{The Alisma and Rhizoma decoction suppresses a-SMA expression in MCD-induced NASH}

We next examined the effect of the Alisma and Rhizoma decoction on HSC in hepatic tissues. Immunohistochemistry revealed increased expression of $\alpha$-SMA, a marker of activated hepatic stellate cells (HSCs), in the hepatic lobule and the sinusoidal space in group II versus group I (Fig. 4), suggesting HSC activation in the liver tissues of mice with MCD-induced NASH-like changes. In group III, $\alpha$-SMA was only expressed scantly in the central vein and portal area, indicating that the Alisma and Rhizoma decoction suppressed $\alpha$-SMA expression in the hepatic tissues of mice with MCD-induced NASH.
The Alisma and Rhizoma decoction inhibits autophagy in hepatocytes in mice with MCD-induced NASH

Recently, autophagy has been implicated in the development and progression of hepatic fibrosis [5-7]. Our transmission electronic microscopy revealed no autophagosomes in the control mice (Fig. 5a). In group II, autophagosomes with bilaminar membranes were present inside the cells and some autophagosomes were fused with lysosomes and formed autophagolysosomes with monolayer membrane (Fig. 5b), suggesting that autophagy was involved in NASH-induced liver injuries. Treatment with the Alisma and Rhizoma decoction markedly reduced the number of autophagosomes, indicating that the decoction may suppress NASH-induced liver fibrosis via inhibition of autophagy (Fig. 5c).

We additionally examined the expression of LC3-II, which correlates with the content of autophagosomes and reflects the activity of autophagosomes. Our immunoblotting assays showed that the Alisma decoction

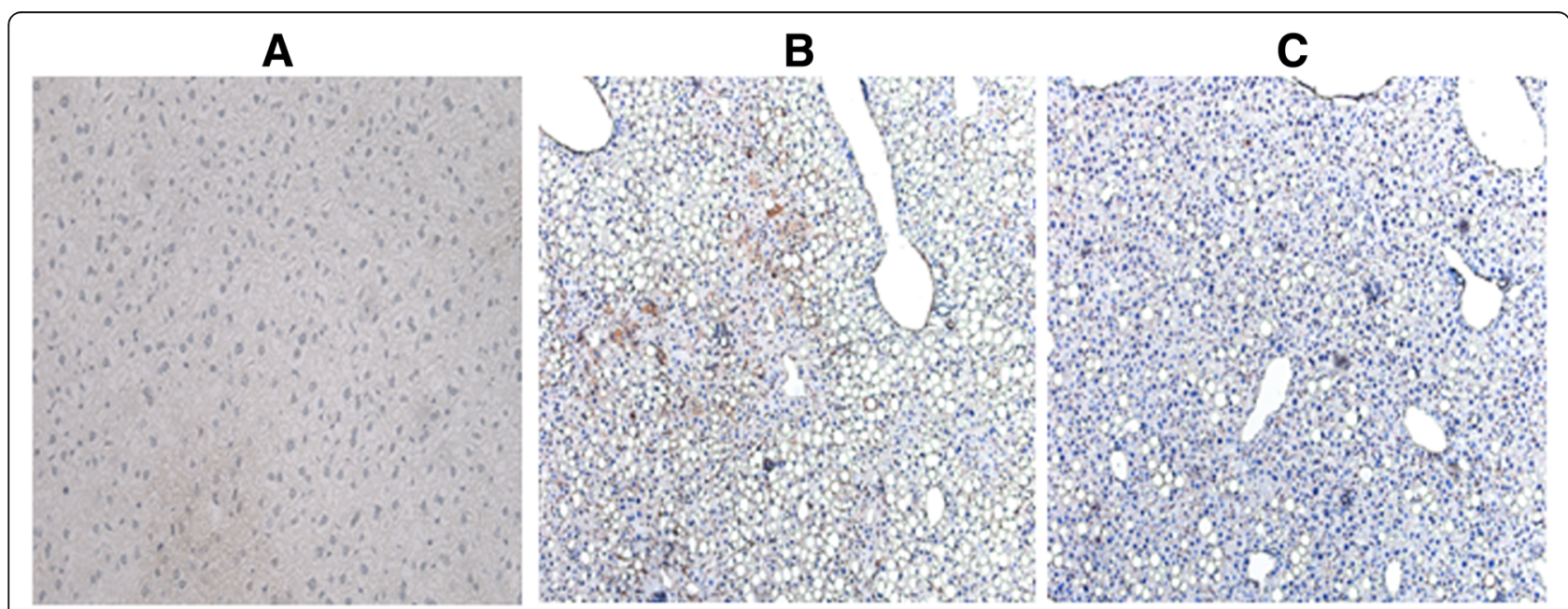

Fig. 4 The Alisma and Rhizoma decoction suppresses a-SMA expression in the hepatic tissues of mice with MCD-induced NASH. The liver tissues of mice receiving regular diet (a), MCD (b) and MCD plus the A. oriental - R. atractylodis macrocephalae decoction (c) were examined by immunohistochemistry for a-SMA. Brown-stained cells are a-SMA positive. Magnification, $100 \times$ 

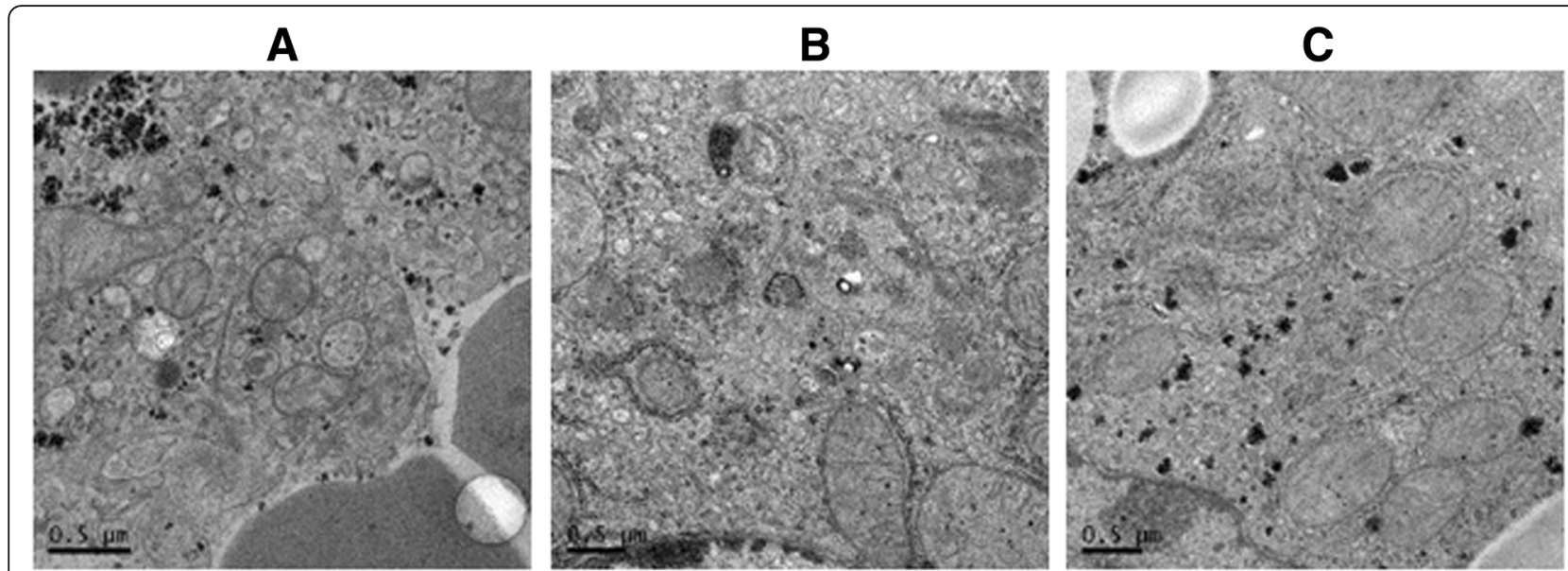

Fig. 5 The Alisma and Rhizoma decoction inhibits autophagy in hepatocytes in mice with MCD-induced NASH. Transmission electronic microscopy reveals no autophagosomes in the control mice (a) while autophagosomes with bilaminar membranes are present inside the cells and some autophagosomes were fused with lysosomes and formed autophagolysosomes with monolayer membrane in group II (b). Treatment with the Alisma decoction markedly reduces the number of autophagosomes (c)

reduced LC3-II expression in hepatic tissues (Fig. 6, the full, uncropped western blots were supplied in the Additional files 1 and 2), suggesting that the decoction reduced the levels of autophagy in hepatic tissues.

\section{Discussion}

The current study demonstrated that the Alisma and Rhizoma decoction lessened NASH-induced liver injuries in mice, which was associated with reduced levels of oxidative stress biomarkers and inhibition of autophagy in hepatic tissues. Inhibition of lipogenesis

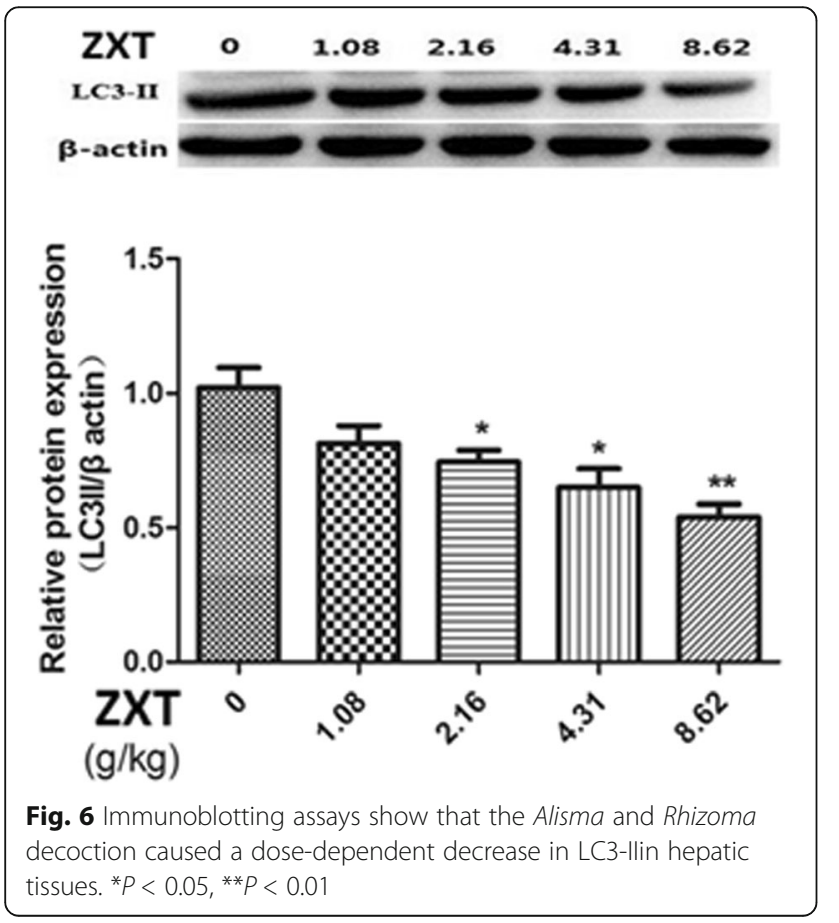

and anti-inflammatory activities have been described for $A$. orientale and $R$. atractylodis macrocephalae as individual medicinal herb $[10,14]$. Though the Alisma and Rhizoma decoction has been shown to possess a myriad of biological and clinical effects, its mechanisms of action have not been elucidated. Our study shed light on the actions of the Alisma and Rhizoma decoction by showing that, apart from inhibition of lipogenesis and anti-inflammatory activities, the Alisma and Rhizoma decoction could alleviate NASH-induced liver injuries by suppressing oxidative stress and autophagy in hepatocytes.

The mechanisms underlying the pathogenesis of NAFLD have not been fully elucidated. It has been hypothesized that fatty liver degeneration as a result of intracellular triglyceride accumulation in hepatocytes represents the primary hit while subsequent oxidative stress and inflammatory response are second hits [27, 28]. Numerous studies have shown that NASH occurs as a result of oxidative stress and mitochondrial injury, which correlates with severity of inflammation in NASH [29-32]. Therapy aiming at oxidative stress may lessen liver inflammation and fibrosis in NASH [33]. In vitro findings indicate that $A$. orientale attenuated long-chain saturated fatty acid-induced cellular injury by inhibiting oxidative stress [26]. We also showed in this study that the Alisma and Rhizoma decoction significantly accentuated the rise in intrahepatocellular SOD activities and suppressed MCD-induced increase in intrahepatocellular MDA contents, suggesting that the Alisma and Rhizoma decoction may alleviate NASH by inhibiting oxidative stress in the liver. In addition, drug delivery and kinetic of phytochemicals in the Alisma and Rhizoma decoction may play an essential role in their therapeutic effects in the liver [34]. 
Oxidative stress and its relevant pathway have a crucial role in liver injury and NAFLD [35]. Oxidative stress may initiate the cascade of HSC activation via the actions of reactive oxidative species (ROS) and lipid peroxidation product such as MDA [36], and activated HSCs are the main culprit of liver fibrosis. We found that the Alisma and Rhizoma decoction downregulated the expression of $\alpha$-SMA, a marker of HSC activation, in hepatic tissues, suggesting that the Alisma and Rhizoma decoction could suppress HSC activation. Autophagy has been shown to be a regulator of lipid metabolism and lipophagy has been proposed to describe the role of autophagy in regulating hepatic lipid metabolism [37]. Recently, it has been shown that autophagy may promote activation and proliferation of HSCs $[8,9,38,39]$. LC3 is a marker of autophagy and LC3-II reflects the activity of autophagy [40]. The current study showed that the Alisma and Rhizoma decoction downregulated the expression of LC3-II in hepatic tissues and reduced the number of autophagosomes, indicating that the Alisma and Rhizoma decoction could inhibit NASH by modulating autophagy.

\section{Conclusion}

In summary, our study has shown that the Alisma and Rhizoma decoction lessens NASH-associated liver injuries by modulating oxidative stress and autophagy in hepatocytes of mice fed with MCD, suggesting that the Alisma and Rhizoma decoction be further explored for its therapeutic action in NAFLD. However, further studies are still required to identify its bioactive constituents, and elucidate the structure-activity relationship and detailed mechanisms of action of the Alisma and Rhizoma decoction.

\section{Additional files}

Additional file 1: The full, uncropped western blots of LC3-II. (TIF $300 \mathrm{~kb}$ ) Additional file 2: The full, uncropped western blots of $\beta$-actin. (TIF $386 \mathrm{~kb}$ )

\section{Abbreviations}

ALT: Alanine aminotransferase; ER: Endoplasmic reticulum; HSC: Hepatic stellate cells; MCD: Methionine and choline diet; NAFLD: Non-alcoholic fatty liver disease; NASH: Non-alcoholic steatohepatitis

\section{Acknowledgements}

None.

\section{Funding}

This work was supported by the National Natural Science Foundation of China (81703879); the Budget Project of Shanghai University of Traditional Chinese Medicine (2016YSN60); Putuo Hospital, Shanghai University of Traditional Chinese Medicine (PTZP201605A, 2016212B). Key specialties of Putuo Hospital Affiliated to Shanghai University of Traditional Chinese Medicine (2016103A). The funding agencies played no role in the design of the study and collection, analysis, and interpretation of data and in writing the manuscript, which are fully the responsibilities of the authors.
Availability of data and materials

The raw data for this study is available upon reasonable request to the corresponding author.

\section{Authors' contributions}

$L X$ and $M J$ performed the experiments and drafted the article. $L Y, L J, P G, J L$, $H L$ and JW helped perform the research, contributed new reagents/analytic tools and analyzed data. QC and YJ designed research, analyzed data, edited and revised the manuscript, and approved the final version of the manuscript. All authors read and approved the final manuscript.

\section{Ethics approval and consent to participate}

The study protocol was approved by the local ethics committee at the authors' affiliated hospital. Animal study was carried out in strict accordance with the established institutional guidelines on the use of experimental animals. Consent to participate was not applicable.

Consent for publication

Not applicable.

\section{Competing interests}

The authors declare that they have no competing interests.

\section{Publisher's Note}

Springer Nature remains neutral with regard to jurisdictional claims in published maps and institutional affiliations.

\section{Author details}

'Department of Gastroenterology, Putuo Hospital, Shanghai University of Traditional Chinese Medicine, Shanghai 200062, China. ${ }^{2}$ Department of Gastroenterology, Shanghai General Hospital/First People's Hospital, School of Medicine, Shanghai Jiao Tong University, Shanghai 201620, China. ${ }^{3}$ Department of Gastroenterology, Shaoxing Hospital of Traditional Chinese Medicine, Shaoxing 312000, China.

Received: 25 October 2018 Accepted: 24 March 2019

Published online: 29 April 2019

\section{References}

1. Koplay M, Sivri M, Erdogan H, Nayman A. Importance of imaging and recent developments in diagnosis of nonalcoholic fatty liver disease. World J Hepatol. 2015;7(5):769-76.

2. Loomba R, Sanyal AJ. The global NAFLD epidemic. Nat Rev Gastroenterol Hepatol. 2013:10(11):686-90.

3. Ji G. Relevant issues concerning clinical trials of traditional Chinese medicine treatment of nonalcoholic fatty liver disease. J Clin Hepatol. 2014; 30(4):299-302.

4. Lee YA, Wallace MC, Friedman SL. Pathobiology of liver fibrosis: a translational success story. Gut. 2015;64(5):830-41.

5. Novo E, Cannito S, Paternostro C, Bocca C, Miglietta A, Parola M. Cellular and molecular mechanisms in liver fibrogenesis. Arch Biochem Biophys. 2014;548:20-37.

6. Wree A, Eguchi A, McGeough MD, Pena CA, Johnson CD, Canbay A, Hoffman HM, Feldstein AE. NLRP3 inflammasome activation results in hepatocyte pyroptosis, liver inflammation, and fibrosis in mice. Hepatology. 2014;59(3):898-910.

7. Tacke F, Trautwein C. Controlling autophagy: a new concept for clearing liver disease. Hepatology. 2011;53(1):356-8.

8. Thoen LF, Guimaraes EL, Dolle L, Mannaerts I, Najimi M, Sokal E, van Grunsven LA. A role for autophagy during hepatic stellate cell activation. J Hepatol. 2011;55(6):1353-60.

9. Hernandez-Gea V, Ghiassi-Nejad Z, Rozenfeld R, Gordon R, Fiel MI, Yue Z, Czaja MJ, Friedman SL. Autophagy releases lipid that promotes fibrogenesis by activated hepatic stellate cells in mice and in human tissues. Gastroenterology. 2012;142(4):938-46.

10. Hong $X$, Tang $H$, Wu L, Li L. Protective effects of the Alisma orientalis extract on the experimental nonalcoholic fatty liver disease. J Pharm Pharmacol. 2006:58(10):1391-8.

11. Zhang LL, Xu W, Xu YL, Chen X, Huang M, Lu JJ. Therapeutic potential of Rhizoma Alismatis: a review on ethnomedicinal application, phytochemistry, pharmacology, and toxicology. Ann N Y Acad Sci. 2017;1401(1):90-101. 
12. Shu Z, Pu J, Chen L, Zhang Y, Rahman K, Qin L, Zheng C. Alisma orientale: Ethnopharmacology, phytochemistry and pharmacology of an important traditional Chinese medicine. Am J Chin Med. 2016;44(2):227-51.

13. Hassan R, Mohammad HF, Khodarahmi R. Polyphenols and their benefits: a review. Int J Food Prop. 2017;20(sup2):1700-41.

14. Jeong HS, Cho YH, Kim KH, Kim Y, Kim KS, Na YC, Park J, Lee IS, Lee JH, Jang HJ. Anti-lipoapoptotic effects of Alisma orientalis extract on non-esterified fatty acid-induced HepG2 cells. BMC Complement Altern Med. 2016;16:239.

15. Meng Q, Duan XP, Wang CY, Liu ZH, Sun PY, Huo XK, Sun HJ, Peng JY, Liu KX. Alisol B 23-acetate protects against non-alcoholic steatohepatitis in mice via farnesoid X receptor activation. Acta Pharmacol Sin. 2017;38(1):69-79.

16. Li CQ, He LC, Dong HY, Jin JQ. Screening for the anti-inflammatory activity of fractions and compounds from Atractylodes macrocephala koidz. J Ethnopharmacol. 2007;114(2):212-7

17. Wang JH, Bose S, Kim HG, Han KS, Kim H. Fermented Rhizoma Atractylodis Macrocephalae alleviates high fat diet-induced obesity in association with regulation of intestinal permeability and microbiota in rats. Sci Rep. 2015;5:8391.

18. Li S, Zhong L, Xia M. Overview of Zexie decoction. Mod Chin Clin Med. 2017;24(4):56-60

19. Wang R, Liu Y, Zhang S. Clinical efficacy and mechanism research of Zexie decoction in the treatment of essential hypertension. Shanghai J Tradit Chin Med. 2017;51(8):103-105+112.

20. Xie J, Wang D. Successful treatment of four cases with classic prescriptions. Hunan J Tradit Chin Med. 2017;33(1):79-81.

21. Park HJ, Han JM, Kim HG, Choi MK, Lee JS, Lee HW, Son CG. Chunggan extract (CGX), methionine-and choline-deficient (MCD) diet-induced hepatosteatosis and oxidative stress in C57BL/6 mice. Hum Exp Toxicol. 2013;32(12):1258-69.

22. Itagaki H, Shimizu K, Morikawa S, Ogawa K, Ezaki T. Morphological and functional characterization of non-alcoholic fatty liver disease induced by a methionine-choline-deficient diet in C57BL/6 mice. Int J Clin Exp Pathol. 2013;6(12):2683-96.

23. Desmet VJ, Gerber M, Hoofnagle JH, Manns M, Scheuer PJ. Classification of chronic hepatitis: diagnosis, grading and staging. Hepatology. 1994;19(6): 1513-20.

24. Ren Z, Li J, Xu N, Zhang J, Song X, Wang X, Gao Z, Jing H, Li S, Zhang C, et al. Anti-hyperlipidemic and antioxidant effects of alkali-extractable mycelia polysaccharides by Pleurotus eryngii var. tuolensis. Carbohydr Polym. 2017; 175:282-92.

25. Xing HC, Li L, Xu KJ, Shen T, Chen YB, Chen Y, Fu SZ, Sheng JF, Chen CL, Wang $J G$, et al. Effects of Salvia miltiorrhiza on intestinal microflora in rats with ischemia/reperfusion liver injury. Hepatobiliary Pancreat Dis Int. 2005;4(2):274-80.

26. Han CW, Kang ES, Ham SA, Woo HJ, Lee JH, Seo HG. Antioxidative effects of Alisma orientale extract in palmitate-induced cellular injury. Pharm Biol. 2012;50(10):1281-8.

27. Jiang ZG, Robson SC, Yao Z. Lipoprotein metabolism in nonalcoholic fatty liver disease. J Biomed Res. 2013;27(1):1-13.

28. Lazebnik LB, Radchenko VG, Golovanova EV, Zvenigorodskaya LA, Konev Y Seliverstov PV, Sitkin SI, Tkachenko El, Aylamazayn EK, Vlasov NN, et al. Nonalcoholic fatty liver disease: diagnostic, symptoms, treatment. Guidelines were approved by the xv gastroenterological scientific society of Russia in 2015. Eksperimental'naia i klinicheskaia gastroenterologiia $=$ Experimental \& clinical gastroenterology. 2015;7:85-96.

29. Gusdon AM, Song KX, Qu S. Nonalcoholic fatty liver disease: pathogenesis and therapeutics from a mitochondria-centric perspective. Oxidative Med Cell Longev. 2014;2014:637027.

30. Nassir F, Ibdah JA. Role of mitochondria in nonalcoholic fatty liver disease. Int J Mol Sci. 2014;15(5):8713-42.

31. Seki E, Brenner DA. Recent advancement of molecular mechanisms of liver fibrosis. J Hepatobiliary Pancreat Sci. 2015;22(7):512-8.

32. De Minicis S, Svegliati-Baroni G. Fibrogenesis in nonalcoholic steatohepatitis Expert Rev Gastroenterol Hepatol. 2011;5(2):179-87.

33. Patsenker E, Schneider V, Ledermann M, Saegesser H, Dorn C, Hellerbrand C, Stickel F. Potent antifibrotic activity of mTOR inhibitors sirolimus and everolimus but not of cyclosporine $A$ and tacrolimus in experimental liver fibrosis. J Hepatol. 2011;55(2):388-98.

34. Zobeiri M, Belwal T, Parvizi F, Naseri R, Farzaei MH, Nabavi SF, Sureda A, Nabavi SM. Naringenin and its nano-formulations for fatty liver: cellular modes of action and clinical perspective. Curr Pharm Biotechnol. 2018;19(3):196-205.
35. Farzaei MH, Zobeiri M, Parvizi F, El-Senduny FF, Marmouzi I, Coy-Barrera E, Naseri R, Nabavi SM, Rahimi R, Abdollahi M. Curcumin in liver diseases: a systematic review of the cellular mechanisms of oxidative stress and clinical perspective. Nutrients. 2018;10(7):855.

36. Apte M. Oxidative stress: does it 'initiate' hepatic stellate cell activation or only 'perpetuate' the process? J Gastroenterol Hepatol. 2002;17(10):1045-8.

37. Zamani M, Taher J, Adeli K. Complex role of autophagy in regulation of hepatic lipid and lipoprotein metabolism. J Biomed Res. 2017;31(5):377-85.

38. Thomes PG, Trambly CS, Thiele GM, Duryee MJ, Fox HS, Haorah J, Donohue TM Jr. Proteasome activity and autophagosome content in liver are reciprocally regulated by ethanol treatment. Biochem Biophys Res Commun. 2012;417(1):262-7.

39. Deng J, Huang Q, Wang Y, Shen P, Guan F, Li J, Huang H, Shi C. Hypoxiainducible factor-1alpha regulates autophagy to activate hepatic stellate cells. Biochem Biophys Res Commun. 2014:454(2):328-34.

40. Li K, Wu D, Chen X, Zhang T, Zhang L, Yi Y, Miao Z, Jin N, Bi X, Wang H, et al. Current and emerging biomarkers of cell death in human disease. Biomed Res Int. 2014;2014:690103.
Ready to submit your research? Choose BMC and benefit from:

- fast, convenient online submission

- thorough peer review by experienced researchers in your field

- rapid publication on acceptance

- support for research data, including large and complex data types

- gold Open Access which fosters wider collaboration and increased citations

- maximum visibility for your research: over $100 \mathrm{M}$ website views per year

At BMC, research is always in progress.

Learn more biomedcentral.com/submissions 